(C) 2020 IEEE. Personal use of this material is permitted. Permission from IEEE must be obtained for all other uses, in any current or future media, including reprinting/republishing this material for advertising or promotional purposes, creating new collective works, for resale or redistribution to servers or lists, or reuse of any copyrighted component of this work in other works. 


\title{
On the Control of Translationally Flexible Base Manipulators
}

\author{
Gianluca Garofalo ${ }^{1}$, Fabian Beck ${ }^{1}$, Julian Klodmann, and Christian Ott
}

\begin{abstract}
This work considers the control of manipulators mounted on a translationally flexible base and extends a previous publication of the authors. The contribution is twofold. Firstly, based on the previously proposed coordinate transformation, a new control strategy is developed, which features two enhancements: The stiffness of the base is allowed to be nonlinear; A momentum-based strategy for vibration damping enables to avoid the feedback linearization, which was required to apply linear stability theory before. Secondly, the control strategies are evaluated in experiments for the first time. Experiments and simulations are used to validate the results and compare the two methods to related approaches.
\end{abstract}

\section{INTRODUCTION}

The control of manipulators on a flexible base was firstly considered in the context of space robotics [1]-[3]. Another well-known application is given by hazardous remoted scenarios, as for example in nuclear power plants [4]. High accuracy and performance requirements for mobile and lightweight robotic systems motivated the study of flexible base manipulators in the context of humanoid robots [5] and in industrial applications [6], [7]. The flexibility of the base causes oscillations that propagate to the end-effector reducing the accuracy of the task execution in inertial frame. In order to cope with the flexible base, various control strategies have been proposed.

Control strategies, which try to avoid base vibrations by appropriate planning of the manipulator trajectory can be categorized as input shaping methods [8], [9]. However, for systems with unknown disturbances or with an initial excitation these kinds of strategies are insufficient [10]. In order to additionally suppress the vibrations once they occur it was proposed to combine input shaping methods with vibration suppression controllers [3], [10]. The control design and stability considerations are often based on singular perturbation theory [11]. For this, it is assumed that the manipulator control and base vibrations are separated in the frequency domain. A different concept to dampen out the base vibrations, while additionally guaranteeing that the manipulator task is fulfilled, is employed in the PseudoPassive Energy Dissipation (P-PED) method [2]. The main idea of this method is to utilize a joint PD controller to achieve the task and to choose the PD control gains such that transfer of energy from the base to the manipulator actuators is maximized. Finally, in the special case of redundant manipulators, the well-known Reaction Null Space

The authors are with the Institute of Robotic and Mechatronics, German Aerospace Center (DLR), Weßling, Germany. ${ }^{1}$ Both authors contributed equally to this work. gianluca.garofalo@dlr.de, fabian.beckedlr.de

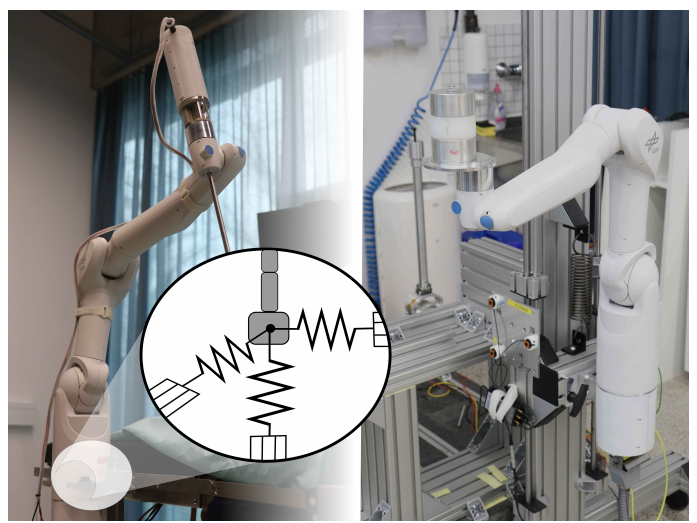

Fig. 1. The considered problem consists of manipulator system on a translationally flexible base. The flexibility of the base is modeled as nonlinear stiffness in all translational directions.

formalism can be applied to flexible base manipulators as proposed in [12].

Very recently, the vibration control problem for manipulators mounted on a translationally flexible and not actuated base was addressed in [13]. The flexibility of the base was assumed to be linear in all three spatial directions, while the orientation of the base is required to be fixed. The study of the translationally flexible base can be regarded as a first step towards the general case with a fully flexible base. Moreover, there might be some applications in which the orientation is stiff or at least stiff enough to have negligible effects, see e.g. [14].

The contribution of this paper is twofold. Firstly, the coordinate transformation proposed in [13] is employed to develop a new control strategy, which features the following enhancements. The stiffness of the base can be nonlinear. In order to further increase the extendability to the fully flexible base, a momentum-based vibration damping strategy is applied. This enables to avoid the feedback linearization of the robot Center of Mass (CoM) dynamics, which was previously required to apply the linear stability theory on the underactuated dynamics. Secondly, both the method of [13] and the new proposed strategy are experimentally verified using a seven Degrees of Freedom (DoF) robot mounted on a one DoF flexible base and compared to other approaches.

The paper is organized as follows. In Section II, the new base vibration strategy is developed for a simplified model. Section III generalizes the method to $n$-link manipulators. In the next Section, three base vibration control strategies are reviewed, which are compared to the proposed strategy in a numerical simulation and in experiments (Section V and VI, respectively). Finally, Section VII concludes the paper. 


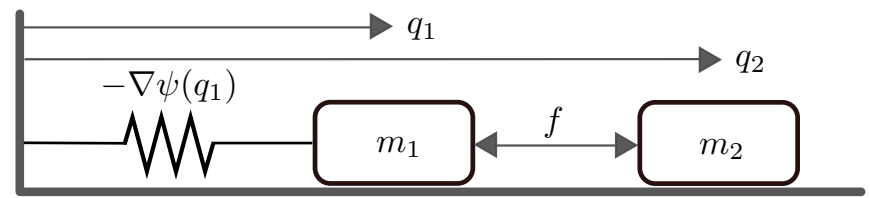

Fig. 2. Sketch of the considered double-mass oscillatory system.

\section{Simplified CASE}

In this section, the vibration control problem is studied using a simple model. Despite its simplicity, this model incorporates many structural properties of the more complex scenario and is used to provide a intuition of the proposed control strategy. The latter is formulated later in Section III.

\section{A. Model}

The model was similarly considered in [2] and [13] and consists of two masses and a spring. A sketch of the system can be found in Fig. 2. The mass $m_{1}$ can be thought of as representing the flexible base, while $m_{2}$ the manipulator attached to it. The quantities $q_{1} \in \mathbb{R}$ and $q_{2} \in \mathbb{R}$ describe the position of the corresponding mass. A nonlinear spring acts on the first mass by exerting the force $-\nabla \psi\left(q_{1}\right)$, where $\psi \in$ $\mathcal{C}^{1}$ is a positive definite function representing the potential energy $\psi\left(q_{1}\right)$ stored in the spring. The force $f \in \mathbb{R}$ is the input of the system, representing an internal force between the two masses. For a control design purpose, the coordinate representing the velocity $\dot{q}_{1}$ of the first mass is replaced by the linear momentum of the system $p=m_{1} \dot{q}_{1}+m_{2} \dot{q}_{2}$. This yields the following dynamic equations of motion

$$
\begin{array}{r}
\dot{q}_{1}=m_{1}^{-1}\left(p-m_{2} \dot{q}_{2}\right), \\
\dot{p}=-\nabla \psi\left(q_{1}\right), \\
m_{2} \ddot{q}_{2}=f,
\end{array}
$$

describing the system in Fig. 2.

\section{B. Control Design}

The control objective is to stop both masses, while additionally steering the second mass to a desired constant equilibrium, i.e., $\dot{q}_{1}, \dot{q}_{2} \rightarrow 0, q_{2} \rightarrow q_{2 d}$ as $t \rightarrow \infty$. This is a challenging task, since only one input is available to satisfy both requirements. A new controller is presented to solve the problem. The underlying idea is to see the system (1) as the interconnection of the subsystem (1a)-(1b) and the subsystem (1c). If $q_{2}$ in (1c) tracks a reference $r_{d}$, then $\dot{r}_{d}$ can be used as a control input for the subsystem (1a)-(1b). The additional requirement $r_{d} \rightarrow q_{2 d}$ is needed to guarantee that $q_{2} \rightarrow q_{2 d}$ as $t \rightarrow \infty$. In order to track the trajectory $r_{d}$ for the second mass, the control action $f$ is chosen as

$$
f=-K e-D \dot{e}+m_{2} \ddot{r}_{d},
$$

where $e=q_{2}-r_{d}$ is the tracking error and $K$ and $D$ are positive constants. This leads to a simple mass-springdamper system for (1c). Assuming $\dot{q}_{2}=\dot{r}_{d}$ to be the input of the subsystems (1a)-(1b), a natural choice to design $\dot{r}_{d}$

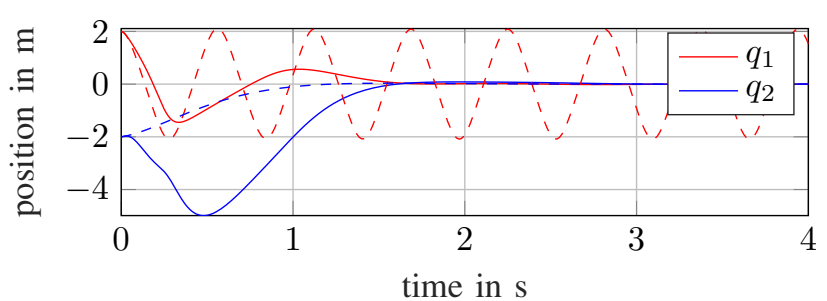

Fig. 3. Response of the system (1) and the control law (2) with the parameters given in Subsection II-C (solid lines). The dashed lines indicate the system behavior without damping strategy, i.e., $K_{p}=0$.

is to consider the energy function $E=\left(2 m_{1}\right)^{-1} p^{2}+\psi\left(q_{1}\right)$ and guarantee that its derivative is nonpositive. The latter is

$$
\dot{E}=\left(-m_{1}^{-1} p+\dot{q}_{1}\right) \nabla \psi\left(q_{1}\right)=-m_{1}^{-1} m_{2} \dot{r}_{d} \nabla \psi\left(q_{1}\right) .
$$

The choice $r_{d}=-K_{p} p+q_{2 d}$, and therefore $\dot{r}_{d}=K_{p} \nabla \psi\left(q_{1}\right)$, leads to $\dot{E}=-m_{1}^{-1} m_{2} K_{p}\left(\nabla \psi\left(q_{1}\right)\right)^{2} \leq 0$. The momentum will go to zero, the mass $q_{1}$ will therefore stop at the equilibrium of the spring and the second mass will reach the desired constant equilibrium $q_{2 d}$, since $r_{d} \rightarrow q_{2 d}$.

\section{Closed-loop Behavior}

The closed-loop dynamics consisting of (1) and (2) yields

$$
\begin{array}{r}
\dot{q}_{1}=m_{1}^{-1}\left(p-m_{2}(\dot{e}+\dot{r})\right), \\
\dot{p}=-\nabla \psi\left(q_{1}\right), \\
m_{2} \ddot{e}+D \dot{e}+K e=0 .
\end{array}
$$

The origin, which is given by $q_{1}=0, p=0$ and $\dot{e}=0, e=$ 0 , is an equilibrium point of the closed-loop. The asymptotic stability of the equilibrium can be shown in three steps using semidefinite Lyapunov functions [15]. For sake of brevity, the proof is omitted as a detailed argumentation is provided in the Section III for the general case. The stability results for the simplified case are summarized in the following theorem.

Theorem 1: Given the system (1) with the control law (2). The origin of the state space is an asymptotically stable equilibrium point of the closed loop (4).

As an example, the control strategy is applied to (1), where the force of the nonlinear stiffness is given by $\nabla \psi\left(q_{1}\right)=30\left(q_{1}+q_{1}^{3}\right)$ and $m_{1}=m_{2}=1$. The gains for the control law (2) are selected to be $K=10, D=5$ and $K_{p}=0.1$. The initial conditions are $q_{1}(0)=2, \dot{q}_{1}(0)=$ $-1, q_{2}(0)=-2, \dot{q}_{2}(0)=1$ and the desired position for the second mass is given by $q_{2 d}=0$. The simulation results are shown in Fig. 3. The developed control law is able to quickly dampen out the vibration in the system, while additionally steering the second mass to the desired position. In comparison to the usual PD controller, i.e the gain $K_{p}$ is set to zero, the additional base damping comes at the price of $q_{2}$ converging not exponentially to the desired equilibrium.

\section{General CASE}

In this section, the control strategy of the simplified case study is generalized to a robotic manipulator. For this, a coordinates transformation is employed, which is along the 
same lines as in [13]. Finally, the stability of the closed loop is analyzed.

\section{A. Model}

The considered systems are n-link manipulators on a flexible base ${ }^{1}$. The flexibility of the base is assumed to be in all three translational directions, while the rotation of the base is considered to be fixed. The stiffness at the base can be nonlinear, as long as it is sufficiently strong to counteract gravity. Let the potential energy of the spring be denoted as $\psi(b) \geq 0$, where $b$ is the position of the base. For a compact configuration manifold, the requirement from above is satisfied if for example

$$
\psi(b) \geq K\|b\|^{2} .
$$

Furthermore, it is additionally assumed, that there exists a homeomorphism identifying $b$ and $\nabla_{b}^{\top} \psi(b)$. The dynamics of the system can be obtained by the Lagrange's equations of motion of the second kind, which yield

$$
M(q) \ddot{q}+C(q, \dot{q}) \dot{q}+g(q)+A \nabla_{b}^{\top} \psi(b)=B \tau,
$$

where $q \in \mathbb{R}^{n+3}$ and $\dot{q} \in \mathbb{R}^{n+3}$ are the generalized coordinates and corresponding velocities. Furthermore, the matrices $M(q), C(q, \dot{q}) \in \mathbb{R}^{(n+3) \times(n+3)}$ denote the positive definite inertia matrix and the Coriolis matrix, respectively. The latter is chosen such that the passivity property, i.e., $\dot{M}(q)=$ $C(q, \dot{q})+C^{\top}(q, \dot{q})$, holds. The vector $g(q) \in \mathbb{R}^{n+3}$ denotes the gravity vector. Having no control input for the base of the manipulator, the vector of generalized torques $\tau \in \mathbb{R}^{n}$ is mapped by a non-square matrix $B \in \mathbb{R}^{(n+3) \times n}$ of rank $n$. The transpose of $B$ maps the generalized velocities to the joint velocities of the manipulator. The force generated by the spring is given by the transposed gradient of the potential $\nabla_{b}^{\top} \psi(b) \in \mathbb{R}^{3}$. The matrix $A \in \mathbb{R}^{(n+3) \times 3}$ maps the external force of the spring to the base, i.e., $A^{\top}$ maps the generalized velocities to the velocity of the base.

\section{B. Control Objective}

The goal for the control action $\tau$ of the manipulator system is to reach a desired task-space configuration $x_{d} \in \mathbb{R}^{n}$ relative to the base, while additionally damping out the vibrations of the elastic base. The task-space coordinate $x_{t}$ (relative to the manipulator's base) is assumed to be given by a diffeomorphism identifying $q$ and $\operatorname{col}\left(b, x_{t}\right)$. Here, $\operatorname{col}(a, \ldots, b)$ denotes the stack of the vectors $a, \ldots, b$. This excludes the case of singular and redundant tasks.

\section{Change of Coordinates}

For the control design and stability analysis, the equations of motion are transformed by a change of coordinates using

$$
\left[\begin{array}{c}
p \\
\dot{r} \\
v
\end{array}\right]=\left[\begin{array}{c}
J_{p}(q) \\
J_{r}(q) \\
N(q)
\end{array}\right] \dot{q}=\left[\begin{array}{c}
J(q) \\
N(q)
\end{array}\right] \dot{q}=T(q) \dot{q},
$$

\footnotetext{
${ }^{1}$ It is assumed that $n \geq 3$. This is necessary to apply the proposed control strategy to manipulators on a flexible base with three translational DoF.
}

where $p \in \mathbb{R}^{3}$ is the linear momentum of the overall system, $r \in \mathbb{R}^{3}$ denotes the CoM of the manipulator (not including the base) expressed in base frame. The definition of the nullspace matrix $N(q)$ is given in the Appendix. As stated in [13], to apply the coordinates transformation two assumptions are needed.

Assumption 1: The Jacobian matrix of the manipulator CoM $J_{r}(q)$ has full row rank for all $q \in \mathbb{R}^{n+3}$.

Assumption 2: It exists a nullspace base matrix $Z(q)$ w.r.t. $J(q)$ satisfying $J(q) Z^{\top}(q)=0 \in \mathbb{R}^{6 \times(n-3)}$, which is differentiable for all $q \in \mathbb{R}^{n+3}$.

Implications and an interpretation of these assumptions can be found in [13]. Analogously to the derivation in [13], the equations of motion of the translationally flexible base manipulator become

$$
\begin{aligned}
{\left[\begin{array}{c}
\dot{p} \\
\Lambda_{r}(q) \ddot{r} \\
\Lambda_{v}(q) \dot{v}
\end{array}\right] } & =-\left[\begin{array}{c}
0 \\
\Gamma_{r}(q, p, \dot{r}, v) \\
\Gamma_{v}(q, p, \dot{r}, v)
\end{array}\right]\left[\begin{array}{c}
p \\
\dot{r} \\
v
\end{array}\right]+\left[\begin{array}{c}
F \\
f \\
u
\end{array}\right], \\
\dot{q} & =J^{+M}(q) \operatorname{col}(p, \dot{r})+Z^{\top}(q) v \\
F & =-m g-\nabla_{b}^{\top} \psi(b),
\end{aligned}
$$

where $J^{+M}(q)=M^{-1}(q) J^{\top}(q)\left(J(q) M^{-1}(q) J^{\top}(q)\right)^{-1}$ is a weighted pseudo-inverse. It is important to note, that the inertial decoupling is achieved by the specific choice of the coordinates. The concepts used to perform the coordinate change can be found in [16]-[18]. The momentum dynamics is determined by the sum of external forces $F \in \mathbb{R}^{3}$, which consists of the constant gravitational force $-m g \in \mathbb{R}^{3}$ and the force $-\nabla_{b}^{\top} \psi(b)$ generated by the spring.

\section{Control Design}

The stabilization of $n$ independent task variables while stopping the base vibration constitutes a challenging control goal, since only $n$ independent control inputs are available in $\tau$. The underactuation of the system becomes obvious in the new coordinates. There is no control input appearing in the momentum dynamics of (8a). The only way to control the momentum is indirectly by affecting the base position via the manipulator. In the following, the control design exploits the connection between base and manipulator given by the momentum of the overall system. The latter is given by the sum of the momentum of the base and of the manipulator,

$$
p=m_{b} \dot{b}+m_{r}(\dot{r}+\dot{b}),
$$

where $m_{b}$ and $m_{r}$ are the mass of the base and the manipulator (excluding its base) with $m_{r}+m_{b}=m$. Solving for the base velocity $\dot{b}$ leads to

$$
\dot{b}=\left(m_{b}+m_{r}\right)^{-1}\left(p-m_{r} \dot{r}\right)
$$

i.e., $\dot{r}$ is related to the base velocity similarly to the relationship in (1a). Therefore, the controller will use a desired velocity reference to stabilize the base and the momentum dynamics, while the robot CoM $r$ is steered to the desired value defined by the task. In the following a physically 
motivated storage function for base and the momentum dynamics is considered, which is given by

$$
V_{s}=\frac{1}{2 m} p^{\top} p+\psi(b)+m g^{\top} b+c .
$$

Here, the constant $c$ is chosen such that $V_{s} \geq 0$ and $V_{s}=0 \Longleftrightarrow p=0, \quad b=b_{d}$. This is possible due to the fact that the stiffness is sufficiently strong to counteract gravity and, by definition, the resting position of the spring at the base $b_{d}$ is the one where $m g=\nabla_{b}^{\top} \psi\left(b_{d}\right)$. The time derivative of $V_{s}$ is given by

$$
\begin{aligned}
\dot{V}_{s} & =m^{-1} p^{\top} F+\nabla_{b} \psi(b) \dot{b}+m g^{\top} \dot{b} \\
& =m^{-1}\left(m_{b} \dot{b}+m_{r}(\dot{r}+\dot{b})\right)^{\top} F-F^{\top} \dot{b} \\
& =\left(m^{-1} m_{r} \dot{r}+\dot{b}\right)^{\top} F-F^{\top} \dot{b} \\
& =m^{-1} m_{r} \dot{r}^{\top} F,
\end{aligned}
$$

where (9) and (8c) as well as $m=m_{r}+m_{b}$ were used. Let $r_{c} \in \mathbb{R}^{3}$ be the desired (constant) reference for the manipulator $\mathrm{CoM}$ in base frame, which is given by the desired task-space configuration $x_{d}$. As in Section II-B, the reference $r_{d}$ of $r$ is chosen as

$$
r_{d}=-K_{p} p+r_{c}
$$

where $K_{p} \in \mathbb{R}^{3 \times 3}$ is a constant positive definite matrix. Assuming that tracking is achieved (i.e., $r=r_{d}$ ), the storage function satisfies $\dot{V}_{s}=-m^{-1} m_{r} F^{\top} K_{p} F \leq 0$. In order to stabilize the trajectory $r_{d}$ for the manipulator CoM, the input $f$ is defined to be

$$
f=\Gamma_{r}(\ldots) \operatorname{col}\left(p, \dot{r}_{d}, v\right)+\Lambda_{r}(q) \ddot{r}_{d}-K_{r} \tilde{r}-D_{r} \dot{\tilde{r}},
$$

where $\tilde{r}=r-r_{d}$ and $K_{r}, D_{r}>0$. As in [13], the potential function for the task-space is given by

$$
U(q)=\frac{1}{2}\left(x_{t}(q)-x_{d}\right)^{\top} K_{x}\left(x_{t}(q)-x_{d}\right)
$$

and it is used in the input $u$

$$
u=\Gamma_{v}(\ldots) \operatorname{col}(p, \dot{r}, 0)-Z(q) \nabla_{q}^{\top} U(q)-D_{v} v,
$$

with $D_{v}>0$.

To apply the control laws (14) and (16) to the robot, the following relationship is employed

$$
T^{\top}(q)\left[\begin{array}{c}
m^{-1} F \\
f \\
u
\end{array}\right]=\left(B \tau-A \nabla_{b}^{\top} \psi(b)-g(q)\right) .
$$

The structure of $T(q)$ assures that $\tau$ is well defined, details on this can be found in the Appendix.

\section{E. Stability Consideration}

The stability properties of the closed-loop system are summarized in the following theorem.

Theorem 2: Given Assumption 1 and Assumption 2, the closed-loop consisting of an $n$-link manipulator on translational base described by (6) with the feedback control law given by (14), (16) and (17) has an asymptotically stable equilibrium point $\chi_{d}$. At this equilibrium point $\chi_{d}$ the

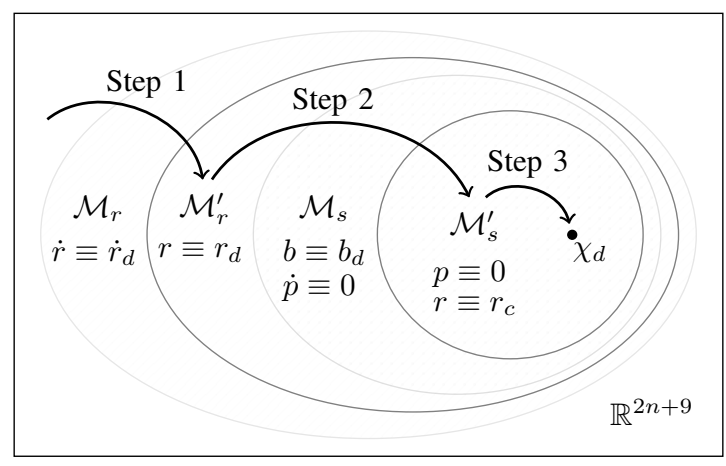

Fig. 4. Sketch of the steps in the proof of Theorem 2. While Step 1 and Step 2 utilize a semidefinite Lyapunov argument of [19], in Step 3 LaSalle's invariance principle is employed to conclude that $v \rightarrow 0$ and $q \rightarrow q_{d}$.

manipulator reaches the desired reference $x_{d}$ in task-space and the base is at rest.

Proof: The closed-loop system is

$$
\begin{aligned}
& \dot{p}=-m g-\nabla_{b} \psi(b), \\
& \Lambda_{r}(q) \ddot{\tilde{r}}+\Gamma_{r r}(\ldots) \dot{\tilde{r}}+D_{r} \dot{\tilde{r}}+K_{r} \tilde{r}=0, \\
& \Lambda_{v}(q) \dot{v}+\Gamma_{v v}(\ldots) v+D_{v} v+Z(q) \nabla_{q}^{\top} U(q)=0, \\
& \dot{q}=J^{+M}(q) \operatorname{col}(p, \dot{r})+Z^{\top}(q) v,
\end{aligned}
$$

where $\Gamma_{r r}(q, p, \dot{r}, v) \in \mathbb{R}^{3 \times 3}$ and $\Gamma_{v v}(q, p, \dot{r}, v) \in$ $\mathbb{R}^{(n-3) \times(n-3)}$ are the corresponding blocks of the Coriolis terms. Let $\chi \in \mathbb{R}^{2 n+9}$ denote the state of (18) and $\chi_{d}$ be the state defined by $p=0, r=r_{c}, \dot{r}=0, v=0$ and $q=q_{d}$. Here, $q_{d}$ is determined by the diffeomorphism using the identification of $q_{d}$ and $\operatorname{col}\left(b_{d}, x_{d}\right)$. It is trivial to show that $\chi_{d}$ is an equilibrium of the closed-loop. The asymptotic stability of $\chi_{d}$ will be shown in three steps, as illustrated in Fig. 4. In the first step, it is shown that the manipulator CoM converges towards the desired trajectory, i.e. $r \rightarrow r_{d}$. In the second step, the storage function $V_{s}$ is used to verify that $b \rightarrow b_{d}, p \rightarrow 0$ and $r_{d} \rightarrow r_{c}$. Finally, it is concluded in Step 3, that $v \rightarrow 0$ and $q \rightarrow q_{d}$.

Step 1: Let $V_{r}$ be the positive semidefinite function ${ }^{2}$

$$
V_{r}(\chi)=\frac{1}{2} \dot{\tilde{r}}^{\top} \Lambda_{r}(q) \dot{\tilde{r}}+\frac{1}{2} \tilde{r}^{\top} K_{r} \tilde{r} .
$$

Using the passivity property for $\Lambda_{r}(q)$ and $\Gamma_{r r}(\ldots)$, the time derivative of $V_{r}$ yields $\dot{V}_{r}(\chi(t))=-\dot{\tilde{r}}^{\top} D_{r} \dot{\tilde{r}} \leq 0$. In the following, a semidefinite Lyapunov argument of [19] is used, which is recalled as Theorem 3 in the Appendix C. In order to apply Theorem 3, one has to show that the equilibrium $\chi_{d}$ of the closed-loop is asymptotically stable on the largest positively invariant set $\mathcal{M}_{r}^{\prime}$ contained in $\mathcal{M}_{r}=$ $\left\{\chi \in \mathbb{R}^{2 n+9}: \dot{V}_{r}(\chi)=0\right\}$. This will be shown by Steps 2-3. For a solution $\chi_{r} \subseteq \mathcal{M}_{r}$ of (18), it holds that $\dot{r} \equiv \dot{r}_{d}$. With (18b) this implies $r \equiv r_{d}$ for $\chi_{r} \subseteq \mathcal{M}_{r}^{\prime}$.

Step 2: The stability argument of Theorem 3 will be employed once more to show that the base and momentum

\footnotetext{
${ }^{2}$ It is important to note, that despite the fact that $r$ is tracking a nonconstant trajectory, $V_{r}$ is time independent as $r_{d}, \dot{r}_{d}$ and $\ddot{r}_{d}$ are functions of the state.
} 
will converge to the desired equilibria if $\chi_{r}$ is contained in $\mathcal{M}_{r}^{\prime}$. For this, the function $V_{s}(\chi) \geq 0$, which was defined in (11) is used. For $\chi_{r} \subseteq \mathcal{M}_{r}^{\prime}$, the time derivative of $V_{s}$ is

$$
\dot{V}_{s}\left(\chi_{r}\right)=\frac{m_{r}}{m}\left(\dot{\tilde{r}}+\dot{r}_{d}\right)^{\top} F=-\frac{m_{r}}{m} F^{\top} K_{p} F \leq 0,
$$

since $\dot{r} \equiv \dot{r}_{d}$ in $\mathcal{M}_{r}^{\prime}$. Thus, one has to show that $\chi_{d}$ is asymptotically stable on the largest positively invariant set contained in $\mathcal{M}_{s}=\left\{\chi \in \mathbb{R}^{2 n+9}: \dot{V}_{s}(\chi)=0\right\} \cap \mathcal{M}_{r}^{\prime}$. A solution $\chi_{s} \subseteq \mathcal{M}_{s}$ satisfies $m g \equiv-\nabla_{b} \psi(b)$, i.e., $b \equiv b_{d}$ since $\dot{p} \equiv F \equiv 0$. Combined with (10) this implies $p \equiv 0$ if $\chi_{s} \subset \mathcal{M}_{s}^{\prime}$. The choice of $r_{d}$ in (13) and $p \equiv 0$ yields $r_{d} \equiv r_{c}$. Additionally, it holds that $r \equiv r_{d}$ for $\chi_{s} \subseteq \mathcal{M}_{s}^{\prime} \subseteq \mathcal{M}_{r}^{\prime}$. In combination this yields $r \equiv r_{c}$ if $\chi_{s} \subseteq \mathcal{M}_{s}^{\prime}$.

Step 3: In this last step, it is shown that $v \rightarrow 0$ and $q \rightarrow q_{d}$ as $t \rightarrow \infty$ for all solutions contained in $\mathcal{M}_{s}$. For this, the function $V_{v}$ and its time derivative given by

$$
\begin{aligned}
& V_{v}(\chi)=\frac{1}{2} v^{\top} \Lambda_{v}(q) v+U(q) \geq 0, \\
& \dot{V}_{v}(\chi)=-v^{\top} D_{v} v \leq 0
\end{aligned}
$$

are utilized. In the derivation of $\dot{V}_{v}$ the passivity property for $\Lambda_{v}(q)$ and $\Gamma_{v v}(\ldots)$ was employed. Using LaSalle's invariance principle [11], it follows that the solution converges into the largest positively invariant subset of $\mathcal{M}_{s}^{\prime}$, which satisfies $v \equiv$ 0 . It follows from $(18 \mathrm{c})$, that a solution contained in this subset fulfills $Z(q) \nabla_{q}^{\top} U(q) \equiv 0$. Since $r \equiv r_{c}$ for $\chi_{s} \subseteq \mathcal{M}_{s}^{\prime}$, this implies $q \rightarrow q_{d}$, which finally yields $\chi_{s} \rightarrow \chi_{d}$. Thus, by Step $2, \chi_{d}$ is asymptotically stable on $\mathcal{M}_{s}^{\prime}$. This completes the proof, as one has obtained that $\chi_{d}$ is asymptotically stable on $\mathcal{M}_{r}^{\prime}$, which was required in Step 1 to conclude asymptotic stability of $\chi_{d}$ for the closed-loop.

\section{OVERVIEW AND COMPARISON OF BASE VIBRATION Control Strategies}

The new proposed approach is validated in simulations and experiments. The method is compared to the classic Cartesian impedance controller, the inertial vibration control proposed in [3] and the approach from [13]. In the following, an overview and a comparison of the methods is given.

\section{A. Cartesian Impedance Control and the P-PED-Method}

The Cartesian impedance control is formulated with respect to the base frame. The control law is given by

$$
\tau_{\text {imp }}=g_{x}(q)-J_{x}(q)^{\top}\left(K_{x}\left(x_{t}-x_{d}\right)+D_{x} J_{x}(q) \dot{q}_{m}\right),
$$

where $g_{x}(q) \in \mathbb{R}^{n}$ is the vector of gravitational torques acting in the robot joints and $J_{x}(q)$ is the Jacobian matrix of $x_{t}$ with respect to the manipulator joints $q_{m}$. In this way, the overall system consisting of manipulator and base is an interconnection of passive subsystems and the term $-D_{x} J_{x}(q) \dot{q}_{m}$ dissipates energy. This is analog to [2]. The disturbance given by the accelerated base will inject energy into the manipulator dynamics. When all the energy will be dissipated, the manipulator and the base will converge towards their desired equilibria. However, for a high taskspace stiffness $K_{x}$, the disturbing effect of the base motion on the manipulator will be small. As a consequence, a desired high task-space stiffness will yield a bad base vibration damping behavior.

\section{B. Inertial Vibration Damping}

In order to dampen out the vibrations of the base, the approach developed in [20] and [3] utilizes inertial forces on the base, which are generated by the manipulator. The implemented control law consists of two terms as follows

$$
\tau=\tau_{\text {imp }}+M_{1}(q) M_{2}^{+}(q) D_{b} \dot{b}
$$

where $M_{1}(q) \in \mathbb{R}^{n \times n}$ and $M_{2}(q) \in \mathbb{R}^{3 \times n}$ are the submatrices of the inertia matrix corresponding to the manipulator inertia and the inertial coupling from manipulator to base, respectively. Compared to [3], here the pseudoinverse, denoted by $(\cdot)^{+}$, was used instead of the inverse of $M_{2}(q)$ since $M_{2}(q)$ is non-square. The first term corresponds to the usual task control defined in (23) and the second term constitutes the additional inertial force counteracting base vibrations. As stated in [3], there are upper bounds for the selection of the gain $D_{b}$. These upper bounds depend on the manipulator configuration $q_{m}$ and are not sufficient to guarantee the stability of the closed loop. Furthermore, the control design is based on the separation of bandwidths for the base damping and the manipulator control, which can not be always guaranteed.

\section{Linear Vibration Damper}

Compared to the previous methods, the approach proposed in [13] requires the full state of the base (position and velocity) and is referred to as Linear Vibration Damper (LVD) in the following. Furthermore, the equilibrium of base $b_{d}$ is assumed to be known. The strategy can be applied to manipulators on a linear translationally flexible base. In the notation of this work, the control law can be stated as

$$
\begin{aligned}
f & =\Gamma_{r}(\ldots) \operatorname{col}(p, \dot{r}, v)+\Lambda_{r}(q) m_{r}^{-1} f_{r}, \\
f_{r} & \left.=-K_{r}\left(r-r_{c}\right)-D_{r} \dot{r}+K_{b}\left(b-b_{d}\right)\right), \\
u & =\Gamma_{v}(\ldots) \operatorname{col}(p, \dot{r}, 0)-Z(q) \nabla_{q}^{\top} U(q)-D_{v} v,
\end{aligned}
$$

where $K_{r}, D_{r}, K_{b} \in \mathbb{R}^{3}$ are positive definite matrices. The feedback laws $f$ and $u$ are applied to the robot as in the proposed approach using (17). In contrast to the inertial vibration control [3], the stability can be always guaranteed given Assumptions 1-2. However, the proof is based on linear stability theory for the vibration damping, therefore the extension to a nonlinear stiffness at the base is not obvious.

\section{Numerical Simulation}

\section{A. Description}

In the considered scenario, the lightweight robot MIRO [21] is simulated on a three DoF flexible base, see Fig. 1 left. The task-space $x_{t}$ for the seven DoF manipulator is given by the six DoF end-effector pose relative to the base and the so called manipulator "elbow", which is given by the third manipulator joint and is denoted by $q_{4}$. The stiffness of the base is chosen to be linear with $2.26 \cdot 10^{3} \mathrm{~N} / \mathrm{m}$ in all spatial directions. The mass of the robot and the base as well as the 
TABLE I

System AND CONTROL PARAMETERS FOR THE MIRO

\begin{tabular}{c|c}
$m_{b}$ & $10.2 \mathrm{~kg}$ \\
\hline$m_{r}$ & $8.2 \mathrm{~kg}$ \\
\hline$K_{\text {pos. }}$ & $600 \mathrm{~N} / \mathrm{m}$ \\
\hline$K_{\text {orient. }}$ & $24 \mathrm{Nm} / \mathrm{rad}$ \\
\hline$K_{\text {elbow }}$ & $18 \mathrm{Nm} / \mathrm{rad}$ \\
IVD and CI \\
$D_{\text {pos }}$ & $100 \mathrm{Ns} / \mathrm{m}$ \\
\hline$D_{\text {orient. }}$ & $5 \mathrm{Nms} / \mathrm{rad}$ \\
\hline$D_{\text {elbow }}$ & $0.5 \mathrm{Nms} / \mathrm{rad}$ \\
\hline$D_{b}$ & $25 \mathrm{Ns}$
\end{tabular}

\begin{tabular}{l|c}
\multicolumn{2}{c}{ LVD } \\
$K_{b}$ & $8000 \mathrm{~N} / \mathrm{m}$ \\
\hline$K_{r}$ & $2000 \mathrm{~N} / \mathrm{m}$ \\
\hline$D_{r}$ & $542.54 \mathrm{~N} / \mathrm{ms}$ \\
\hline$D_{n}$ & $25 \mathrm{~N} / \mathrm{ms}$ \\
Proposed Approach \\
$K_{r}$ & $10000 \mathrm{~N} / \mathrm{m}$ \\
\hline$D_{r}$ & $1100 \mathrm{Ns} / \mathrm{m}$ \\
\hline$D_{n}$ & $25 \mathrm{~N} / \mathrm{ms}$ \\
\hline$K_{p}$ & $0.0081 / \mathrm{s}$
\end{tabular}

chosen control gains can be found in Table I. For comparison, the task-space stiffness $K_{x}=\operatorname{diag}\left(K_{\text {pos }} I_{3}, K_{\text {orient. }} I_{3}, K_{\text {elbow }}\right)$ was selected to be the same for all approaches. Having fixed the stiffness, the damping gain $D_{x}$ constitutes the only design parameter for the Cartesian impedance controller. The damping parameters are specified in subtable "IVD and CI", using the same structure of notation as for $K_{x}$.

After five seconds of simulation, the periodic disturbance

$$
d_{z}(t)=\left\{\begin{array}{ll}
0 & t \leq 5 \\
10 N \sin \left(\sqrt{\frac{2260 \mathrm{~kg} / \mathrm{s}}{m_{c}}} t\right) & t>5
\end{array},\right.
$$

is acting along the $z$-direction of the end-effector.

\section{B. Discussion of the Results}

The simulation results are reported in Fig. 5 and Fig. 6. In the considered case, the gain tuning for the inertial vibration control strategy was very challenging. This was due to the fact, that the base and the manipulator oscillate in the same frequency bandwidth. Therefore, the separation of the two time scale control does not hold and the vibration damping control action may even cause instability. In other simulation scenarios, the performance of the inertial vibration damping method was acceptable and the gain tuning was straightforward. Another difficulty in the implementation of the inertial vibration damping is that the gain tuning is configuration dependent. Compared to the Cartesian impedance controller and the inertial vibration damping, the LVD and the proposed approach achieve a better vibration damping performance. Another important difference can be observed with respect to the response of the disturbance. The simulation clearly shows, that for the proposed approach and LVD, the disturbance effects only the base in the perturbed direction. Additionally, the error in task-space is mainly in $z$ direction for both methods. These two decoupling features are not observable for the other approaches.

\section{EXPERIMENTAL RESULTS}

\section{A. Description of the Setup}

The experimental setup consists of the lightweight robot MIRO, which is attached to a vertical linear guidance as base. The setup is shown in Fig. 1 (right). The flexibility of the base is realized along the linear track using a spring, which is preloaded by the gravitational force. Near the operation point, the stiffness of the spring is estimated to be $2.26 \cdot 10^{3} \mathrm{~N} / \mathrm{m}$. The mass of the robot and the base are
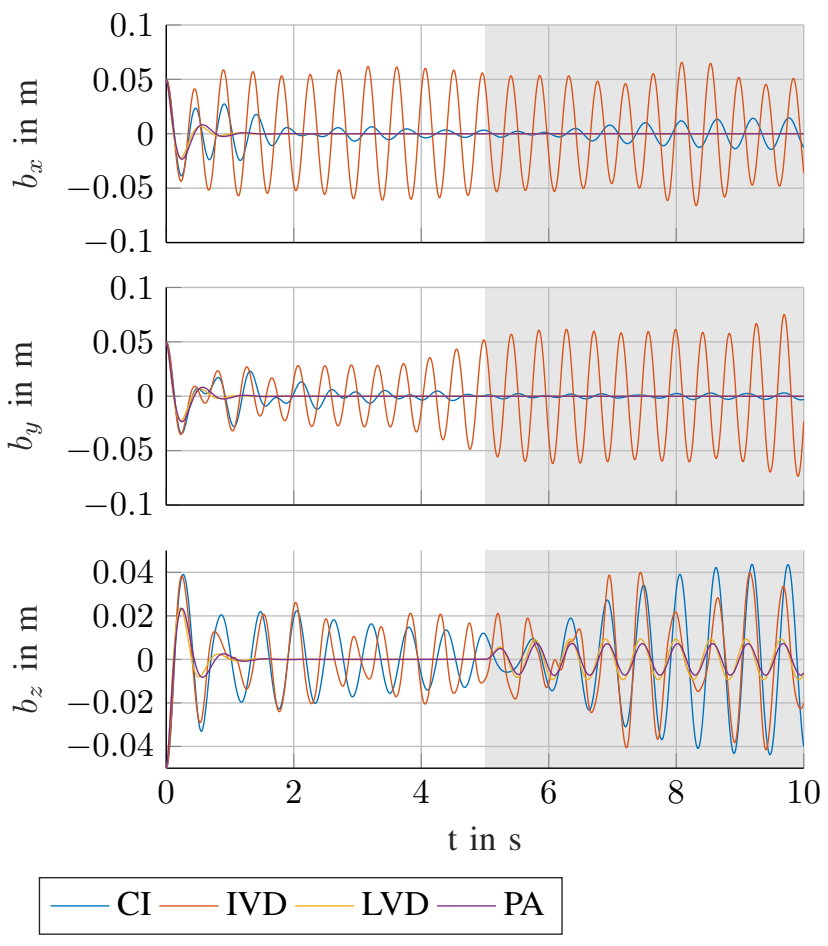

Fig. 5. Plot of the base position $b$ for $x-, y-$ and $z$-direction obtained in the numerical simulation.
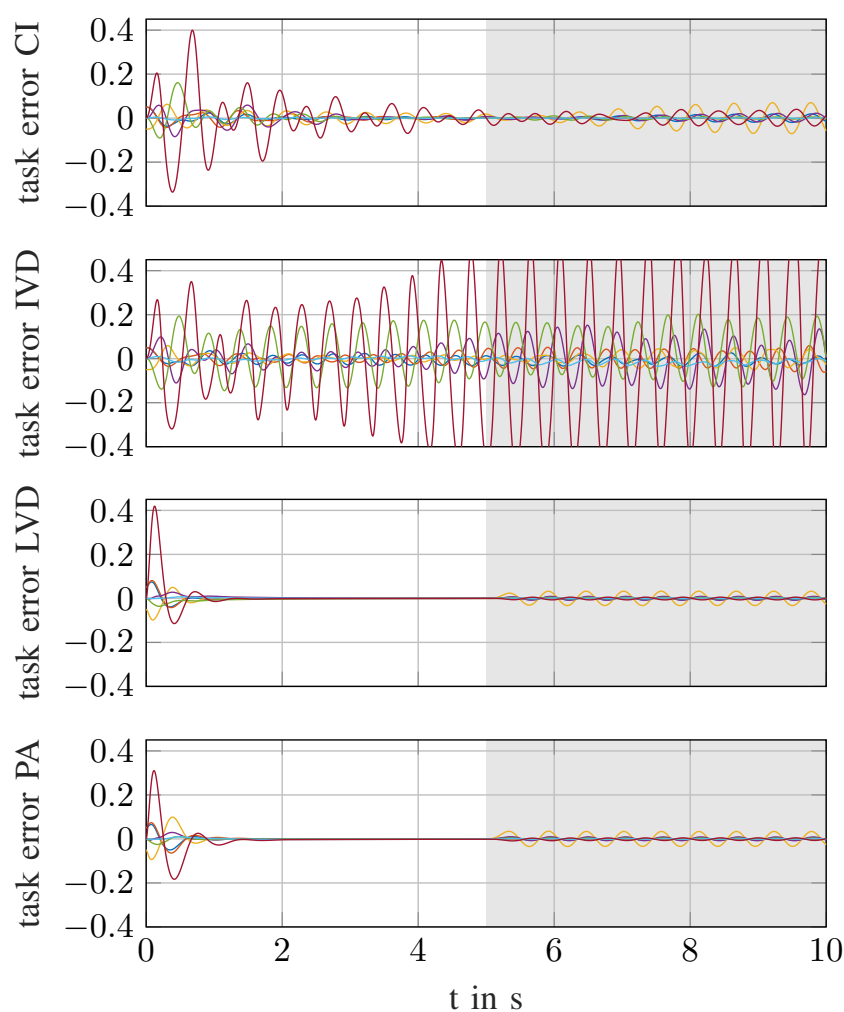

$$
-\mathrm{x}-\mathrm{y}-\mathrm{z}-\alpha-\beta-\gamma-\tilde{q}_{4}
$$

Fig. 6. Task-space error obtained in the simulation in inertial frame for the classical Cartesian impedance (CI) controller, inertial vibration damping (IVD) method, the Linear Vibration Damper (LVD) and the proposed approach (PA). Here, $x, y, z$ and $\alpha, \beta, \gamma$ denote, the position error in $\mathrm{m}$ and the quaternion error, respectively. The robot elbow error in rad is denoted by $\tilde{q}_{4}$. 
TABLE II

CONTROL PARAMETERS IN THE EXPERIMENTS

\begin{tabular}{|c|c|c|c|c|c|}
\hline \multicolumn{2}{|r|}{ LVD } & \multicolumn{2}{|c|}{ IVD and CI } & \multicolumn{2}{|c|}{ Proposed Approach } \\
\hline$K_{b}$ & $720 \mathrm{~N} / \mathrm{m}$ & $D_{\text {pos }}$ & $10 \mathrm{Ns} / \mathrm{m}$ & $K_{r}$ & $10000 \mathrm{~N} / \mathrm{m}$ \\
\hline$K_{r}$ & $2000 \mathrm{~N} / \mathrm{m}$ & $D_{\text {orient. }}$ & $0.5 \mathrm{Nms} / \mathrm{rad}$ & $D_{r}$ & $1100 \mathrm{Ns} / \mathrm{m}$ \\
\hline$D_{r}$ & $542.54 \mathrm{~N} / \mathrm{ms}$ & $D_{\text {elbow }}$ & $0.05 \mathrm{Nms} / \mathrm{rad}$ & $D_{n}$ & $2.5 \mathrm{~N} / \mathrm{ms}$ \\
\hline$D_{n}$ & $2.5 \mathrm{~N} / \mathrm{ms}$ & $D_{b}$ & $25 \mathrm{Ns}$ & $K_{p}$ & $8 \cdot 10^{-4} 1 / \mathrm{s}$ \\
\hline
\end{tabular}

the same as in the simulation (see Table I). The position of the base is measured by a camera detecting a marker attached to the base of the robot. The position measurements are provided at a frequency of $1 \mathrm{kHz}$. When necessary, the velocity of the base was estimated by a Kalman filter using the base position measurements. The robot MIRO is commanded using a joint torque interface at a frequency of $3 \mathrm{kHz}$. Compared to the model in (6) and the numerical simulation, the experimental setup has viscose friction and stiction due to the linear guidance. These additional effects act as natural base vibration damping and are assumed to be a model uncertainty.

\section{B. Design of the Experiment}

In the beginning of the experiment, the robot is at its desired configuration in task-space. Whereas, the base is deflected by about $-7.5 \mathrm{~cm}$ using a weight attached to the base by a rope. By cutting the rope, the spring accelerates the base upwards. The controller is enabled as soon as the base passes $-7.0 \mathrm{~cm}$. The task-space $x_{t}$ is chosen as in Section V. The control objective was to rapidly dampen out the base vibrations while achieving the desired task. A second criterion for tuning the gains was that the manipulator should implement a reasonable stiffness to obtain a good disturbance rejection and allows for human interaction. As in the numerical simulation, for comparison, the task-space stiffness $K_{x}$ was selected to be the same for all approaches (see Table I). All other control gains are specified in the corresponding subtables of Table II. For the inertial vibration control strategy, the gain $D_{b}$ was increased from zero until manipulator limitations were reached. In this experiment, the best base damping behavior was achieved with the highest damping gain, which is reported in Table II. The new developed strategy requires the momentum and its higher derivatives as reference in the control law. These quantities were estimated using a Kalman filter.

\section{Discussion of the Results}

The results of the experiments are reported in Fig. 7. Additionally, a video is available [22]. For all approaches, the base vibration is decreasing over time. As expected, the worst result for the vibration damping of the base was achieved by the Cartesian impedance controller, as the base settled after 2.5s. The IVD and LVD method achieved a settling time of about $1.8 \mathrm{~s}$. While the proposed approach stopped the base in less than $1.5 \mathrm{~s}$. The faster convergence for the proposed approach comes at the price of a higher end-effector deflection in the transition.

In the experiments the control parameters of the simulation had to be adapted due to actuator limitations and friction.

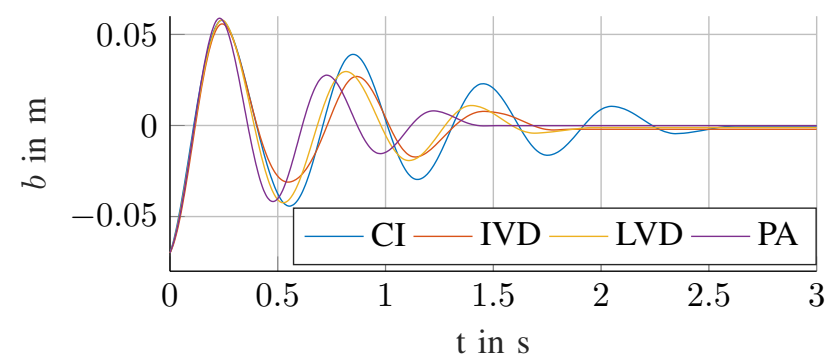

Fig. 7. Base position $b$ obtained in the experiments for the classical Cartesian impedance (CI) controller, inertial vibration damping (IVD) method, the Linear Vibration Damper (LVD) and the proposed approach (PA).

For the LVD, $K_{b}$ increases the closed-loop stiffness at the base and is related to the base vibration damping [13]. While for the proposed approach, an increase of $K_{p}$ yields a better damping of the base motion, see (20). For both, the proposed approach and LVD, these gains are reduced compared to the simulation. Therefore, vibration damping performance can not be as effective as before. Compared to the simulation, the performance of the IVD and Cartesian Impedance controller was significantly improved. Both methods took full advantage of the friction present in the experimental setup. While, for LVD and the proposed approach, the friction did not have such a significant impact.

\section{CONCLUSION}

In this paper, it was considered the problem of regulating a robotic manipulator mounted on a translationally flexible base to a desired configuration. The designed controller is able to guarantee the requirement while simultaneously stop the motion of the base. Compared to the previous version of the control law several improvements have been obtained and additionally experimental validation of both approaches has been demonstrated. The new method avoids feedback linearization and can cope with nonlinear springs. This is possible by leveraging on first principles of mechanics, in particular the connection between the dynamics of the linear momentum and the external forces. In this way the design of the controller does not need to rely on eigenvalue analysis. The importance of using the momentum relies as well on the possibility to consider the method for future extensions addressing the rotational case. For this case, the use of the angular momentum seems promising for the solution of the more general problem.

\section{APPENDIX}

For completeness, the content of Subsection A and B is taken from [13] and shows the expressions of the nullspace projector $N$ and of the velocity map $T$. All the matrices are partitioned accordingly to the state $q=\operatorname{col}\left(b, q_{m}\right)$, where $q_{m} \in \mathbb{R}^{n}$ are the generalized coordinates corresponding to the manipulator. The dependencies are dropped for sake of brevity. Subsection $\mathrm{C}$ recalls the stability theorem using semidefinite Lyapunov functions of [19] employed in the proof of Theorem 2. 


\section{A. Nullspace projector computation}

Let $M_{m}$ be the inertia matrix of the manipulator, when the base coordinates are replaced with the overall CoM. This is done as in [23], by using the Jacobian matrix $J_{c}$ of the overall CoM (including the base) to block-diagonalize the inertia matrix $M$. In this way, $M_{m}$ is the block appearing on the diagonal in correspondence of the manipulator coordinates.

The Jacobian matrix $J_{r}$ can be written as $J_{r}=$ $\left[\begin{array}{ll}O_{3} & J_{r, m}\end{array}\right]$, with $O_{3} \in \mathbb{R}^{3 \times 3}$ a matrix of zeros and $J_{r, m} \in \mathbb{R}^{3 \times n}$. At this point, a nullspace base matrix [18] $Z_{r, m} \in \mathbb{R}^{(n-3) \times n}$ can be computed, such that $\forall q_{m} \in \mathbb{R}^{n}$ : $J_{r, m} Z_{r, m}^{\top}=0$. Given $Z_{r, m}$, one can compute

$$
N_{r, m}=\left(Z_{r, m} M_{m} Z_{r, m}^{\top}\right)^{-1} Z_{r, m} M_{m},
$$

so that finally $N=\left[\begin{array}{ll}O_{n-3,3} & N_{r, m}\end{array}\right]$, where $O_{n-3,3} \in$ $\mathbb{R}^{n-3 \times 3}$ is a matrix of zeros. Partitioning the matrices in blocks, it is possible to verifythat $N=\left[\begin{array}{ll}O_{n-3,3} & N_{r, m}\end{array}\right]$ is a dynamically consistent nullspace projector [18].

\section{B. Input Transformation}

Given the introduced matrix partitioning and the expression of $N$, the velocity map $T$ has the expression

$$
T=\left[\begin{array}{cc}
m I_{3} & m J_{c, m} \\
O_{3} & J_{r, m} \\
O_{n-3,3} & N_{r, m}
\end{array}\right],
$$

with $J_{c, m} \in \mathbb{R}^{3 \times n}$ being the manipulator block of the overall-CoM Jacobian matrix $J_{c}$ and $I_{3}$ the identity matrix. Notice that the relationship $J_{p}=m J_{c}$ has been used. With this, the first three equations of (17) are always satisfied and the remaining $n$ equations can be rewritten as

$$
\begin{aligned}
& J_{c, m}^{\top}\left(-m g-\nabla_{b}^{\top} \psi(b)\right)+J_{r, m}^{\top} f+N_{r, m}^{\top} u \\
& \quad+\left[\begin{array}{ll}
O_{n, 3} & I_{n}
\end{array}\right]\left(A \nabla_{b}^{\top} \psi(b)+g\right)=B_{m} \tau .
\end{aligned}
$$

Since $B_{m} \in \mathbb{R}^{n \times n}$ is invertible, it is always possible to compute the torques $\tau$ that solve the control problem.

\section{Stability with Semidefinite Lyapunov Functions}

Theorem 3 (Theorem 2 of [19]): Let $\chi_{0}$ be an equilibrium point for the dynamical system $\dot{\chi}=f(\chi)$. If in a neighborhood $U$ of $\chi_{0}$ there exists a $\mathcal{C}^{1}$ function $V: U \rightarrow \mathbb{R}$ such that $V(\chi) \geq 0$ and $\dot{V}(\chi) \leq 0$ for all $\chi \in U$ and $V\left(\chi_{0}\right)=0$ and $\chi_{d}$ is asymptotically stable on the largest positively invariant set contained in $\{\chi \in U: \dot{V}(\chi)=0\}$ then $\chi_{0}$ is an asymptotically stable equilibrium point for $\dot{\chi}=f(\chi)$.

\section{REFERENCES}

[1] A. Sharon and D. Hardt, "Enhancement of robot accuracy using endpoint feedback and a macro-micro manipulator system," in American Control Conference, June 1984, pp. 1836-1845.

[2] M. A. Torres, S. Dubowsky, and A. C. Pisoni, "Vibration control of deployment structures' long-reach space manipulators: The PPED method," in Proceedings of IEEE International Conference on Robotics and Automation, vol. 3, 1996, pp. 2498-2504.

[3] L. E. George and W. J. Book, "Inertial vibration damping control of a flexible base manipulator," IEEE/ASME Transactions on Mechatronics, vol. 8, no. 2, pp. 268-271, June 2003.
[4] C. Ha, H. Kim, and D. Lee, "Experimental evaluation of passivitybased control of manipulator-stage system on flexible beam," in 14th International Conference on Ubiquitous Robots and Ambient Intelligence (URAI), 2017, pp. 465-466.

[5] T. Wimböck, D. Nenchev, A. Albu-Schäffer, and G. Hirzinger, "Experimental study on dynamic reactionless motions with DLR's humanoid robot Justin," in IEEE/RSJ International Conference on Intelligent Robots and Systems, Oct 2009, pp. 5481-5486.

[6] J. Y. Lew and S. M. Moon, "A simple active damping control for compliant base manipulators," IEEE/ASME Transactions on Mechatronics, vol. 6, no. 3, pp. 305-310, Sep 2001.

[7] C. Ott, A. Albu-Schaffer, and G. Hirzinger, "A cartesian compliance controller for a manipulator mounted on a flexible structure," in IEEE/RSJ International Conference on Intelligent Robots and Systems, Oct 2006, pp. 4502-4508.

[8] M. A. Torres and S. Dubowsky, "Path-planning for elastically constrained space manipulator systems," in Proceedings IEEE International Conference on Robotics and Automation, vol. 1, May 1993, pp. 812-817.

[9] N. C. Singer and W. P. Seering, "Preshaping command inputs to reduce system vibration," ASME Journal of Dynamic Systems, Measurement, and Control, vol. 112, pp. 76-82, 1990.

[10] D. P. Magee, D. W. Cannon, and W. J. Book, "Combined command shaping and inertial damping for flexure control," in Proceedings of the American Control Conference (Cat. No.97CH36041), vol. 3, June 1997, pp. 1330-1334.

[11] H. K. Khalil, Nonlinear Systems. New Jersey: Prentice Hall, 2002.

[12] D. N. Nenchev, K. Yoshida, and M. Uchiyama, "Reaction null-space based control of flexible structure mounted manipulator systems," in Proceedings of IEEE Conference on Decision and Control, vol. 4, Dec 1996, pp. 4118-4123.

[13] F. Beck, G. Garofalo, and C. Ott, "Vibration control for manipulators on a translationally flexible base," in IEEE Int. Conf. on Robotics and Automation (ICRA), Montreal, Canada, 2019, pp. 4451-4457.

[14] J. Y. Lew and S.-M. Moon, "Acceleration feedback control of compliant base manipulators," in Proceedings of the American Control Conference, vol. 3, 1999, pp. 1955-1959.

[15] A. Iggidr, B. Kalitine, and R. Outbib, "Semidefinite Lyapunov functions: Stability and stabilization," in Mathematics of Control, Signals, and Systems, 1996, pp. 95-106.

[16] O. Khatib, "A unified approach for motion and force control of robot manipulators: The operational space formulation," IEEE Journal on Robotics and Automation, vol. 3, no. 1, pp. 43-53, February 1987.

[17] J. Park, W. Chung, and Y. Youm, "On dynamical decoupling of kinematically redundant manipulators," in IEEE/RSJ International Conference on Intelligent Robots and Systems, 1999, pp. 1495-1500.

[18] C. Ott, Cartesian Impedance Control of Redundant and Flexible-Joint Robots, ser. Springer Tracts in Advanced Robotics. Berlin: SpringerVerlag, 2008.

[19] A. Iggidr, B. Kalitine, and G. Sallet, "Lyapunov theorems with semidefinite functions," IFAC Proceedings Volumes, vol. 32, no. 2 pp. 1802 - 1807, 1999, 14th IFAC World Congress

[20] W. J. Book and S. H. Lee, "Vibration control of a large flexible manipulator by a small robotic arm," in American Control Conference, June 1989, pp. 1377-1380

[21] U. Hagn, M. Nickl, S. Jörg, G. Passig, T. Bahls, A. Nothhelfer, et al., "The DLR MIRO: a versatile lightweight robot for surgical applications," Industrial Robot, pp. 324-336, 2008.

[22] Video experiments, https://dlrmax.dlr.de/get/835bbd43-2ad7-5593b15f-6f3096538610/

[23] G. Garofalo, B. Henze, J. Englsberger, and C. Ott, "On the inertially decoupled structure of the floating base robot dynamics," in International Conference on Mathematical Modelling, Vienna, Austria, Feb. 2015, pp. 322-327. 